\title{
Desenvolvimento de Elastômeros Termoplásticos Vulcanizados (TPV) a Base de Polipropileno com Resíduo de Pneu. I - Planejamento Fatorial de Experimentos
}

\author{
Matheus M. Cossa, Alex S. Sirqueira \\ Centro Universitário Estadual da Zona Oeste, RJ \\ Bluma G. Soares \\ Instituto de Macromoléculas Professora Eloisa Mano, UFRJ
}

Resumo: O uso do pó de pneu em misturas com termoplásticos possibilita a formação de novos materiais. Nesse trabalho o uso do pó de pneu foi caracterizado pelo planejamento fatorial completo $2^{3}$, com ponto central, em misturas com polipropileno (PP), para desenvolvimento de novos elastômeros termoplásticos vulcanizados dinamicamente (TPVs), contendo material reciclado. Os fatores utilizados neste estudo foram, pó de pneu, peróxido de dicumila e bismaleimida (em três níveis, sendo um deles, o ponto central). O planejamento foi construído para avaliar os efeitos dos fatores (principais e suas interações) nas respostas de tração, alongamento e inchamento em óleo. Os resultados mostraram que o teor de pó de pneu é predominante para melhorar o desempenho das misturas. A resistência ao inchamento é aproximadamente $150 \%$ superior em algumas formulações.

Palavras-chave: Pó de pneu, reciclagem, elastômeros termoplásticos, PP e SBR.

\section{Development of Thermoplastic Elastomers Vulcanized (TPV) with Polypropylene Waste Tire. I - Factorial Design Experiments}

\begin{abstract}
The use of waste tire in blends with thermoplastics enables the formation of new materials. In this paper the use of waste tire was characterized with a complete $2^{3}$ factorial design with central point, in blends with polypropylene (PP), in the development of new thermoplastic elastomers vulcanized dynamically (TPVs) and containing recycled material. The factors used in this study were: waste tire, dicumylperoxide and bismaleimides in three levels, one being the central point. The factorial design was built to evaluate the effects of the factors (and their interaction) in the responses of tensile, elongation and oil swelling. The results showed that the level of waste tire is predominant to improve the performance of TPV. The swelling resistance is approximately $150 \%$ higher in some formulations.
\end{abstract}

Keywords: Waste tire, recyclable, thermoplastic elastomeric, PP and SBR.

\section{Introdução}

O uso de material reciclado em novas aplicações é uma alternativa viável para reduzir a quantidade de lixo despejado no meio ambiente. Dentre os materiais poliméricos mais nocivos destacam-se aqueles que contem cargas de metais pesados, plastificantes e os pneus. Estes por serem reticulados e não reprocessáveis, são os mais indesejados, devido ao elevado tempo de degradação e a problemas de saúde pública como a dengue ${ }^{[1,2]}$. Os pneus fabricados pelas indústrias representam um prejuízo ambiental estimado em 100 milhões de pneus no Brasil. As tecologias mais empregadas hoje, para a retutilização dos pneus são: recauchutagem, regeneração, reciclagem, reciclagem energética, pirólise, adição em formulação de asfaltos, além de diversas outras formas de reutilização ${ }^{[3]}$. O uso do pó de pneu em misturas com termoplásticos tem ampla aplicação tecnológica e científica, porque são capazes de produzirem novos materiais com facilidade de processamento e boas propriedades elásticas. Entretanto, devido às diferenças estruturais e de viscosidade, a simples adição dos dois compostos não produz um novo material com sinergismo de propriedades ${ }^{[4-6]}$. É freqüente o uso de aditivos especiais a fim de compatibilizar e, consequentemente melhorar o desempenho da mistura. O que em muitos casos pode inviabilizar economicamente a produção, devido ao elevado custo dos compatibilizantes. A literatura reporta o uso de elastômero virgem como agente redutor da tensão interfacial entre o termoplástico e o elastômero ${ }^{[7-10]}$. O comportamento mecânico dos elastômeros termoplásticos vulcanizados está conexo com o sistema de reticulação es-

Autor para correspondência: Alex S. Sirqueira, Centro Universitário Estadual da Zona Oeste, Av. Manuel Caldeira de Alvarenga 1203 - Campo Grande, CEP: 23070-200, Rio de Janeiro, RJ. Brasil.E-mail: asirqueira@click21.com.br 
colhido, pois o desempenho da mistura é função dos tipos de ligações químicas produzidas ${ }^{[9]}$.

Nesse trabalho foi avaliado o uso do pó de pneu em compostos com polipropileno, para obter um novo elastômero termoplástico vulcanizado dinamicamente (TPV) com material reciclado. Nessa etapa do estudo utilizou-se o planejamento fatorial de experimentos a fim de avaliar quais variáveis da formulação são mais relevantes e a resposta nas propriedades mecânicas do TPV.

Para reticular a fase elastomérica foi utilizado o sistema de cura a base de peróxido orgânico e co-agente de reticulação. O copolímero de estireno-butadieno (SBR) foi adicionado na mistura para avaliar há necessidade do uso de material virgem na composição como compatibilizante do sistema Polipropileno e pó de pneu.

\section{Metodologia}

\section{Materiais}

Homopolímero de Polipropileno (PP) com índice de fluidez de 3,5 g/10 min. produzido pela Braskem. Copolímero de estireno-butadieno (SBR1502), com teor de estireno combinado de 23,5\% e viscosidade Mooney MML $\left(1+4 @ 100{ }^{\circ} \mathrm{C}\right)=52$, produzido pela Petroflex Ind. Com. Resíduo de pneu sob a forma de pó cedido pela Itaipava recauchutora S/A, com diâmetro médio de partículas de 35 mesh; Peróxido de dicumila cedido pela Retilox Ind. Com. com grau de pureza de $99 \%$ e o co-agente 1,1-(trimetilenodi-4,1 fenileno) bismaleimida (BMI) com grau de pureza comercial cedido pela Cya rubber.

\section{Composição das misturas}

As misturas foram processadas em misturador interno Brabender com velocidade de rotação de 80 RPM, rotores do tipo Banbury e tempo total de processamento de 8 minutos. Inicialmente o PP foi processado e após 2 minutos adicionouse a fase elastomérica (pó de pneu e/ou SBR) até estabilização do torque de mistura. Após esse período foi adicionado o sistema de reticulação composto pelo co-agente e pelo peróxido de dicumila, deixou-se reagir por mais 2 minutos. Fixou-se a composição das misturas em $30 \mathrm{phr}$ da fase termoplástica e $70 \mathrm{phr}$ da fase elastomérica. Essa etapa do estudo fez-se somente a variação na fração elastomérica do TPV, esta estratégia foi adotada para obter um elastômero termoplástico vulcanizado com maior caráter elástico. Após o processamento as massas foram pesadas para verificar a eficiência da mistura, adotou-se $2 \%$ como variação aceitável.

Os compostos foram injetados em mini-injetora de bancada (MINIJET - Haake) a $240{ }^{\circ} \mathrm{C}$ sob pressão de 300 bar e pressão de recalque de 150 bar por 5 segundos. As medidas de tração foram determinadas em Máquina Universal de Ensaios Instron modelo 5569, com velocidade de separação entre as garras de $100 \mathrm{~mm} / \mathrm{min}$ de acordo com a norma ASTM D 638-02 para corpos de prova do tipo IV. O en- saio de inchamento em óleo foi realizado com óleo de motor (Petrobras SJ 20/50) na temperatura de $80{ }^{\circ} \mathrm{C}$ durante 72 horas, de acordo com a norma ASTM D3616-95.

Os dados de resposta foram analisados estatisticamente, utilizando um modelo fatorial $2^{3} \mathrm{com}$ um ponto central, com auxílio do programa MINITAB $13^{[11]}$. Os resultados do planejamento fatorial podem ser expressos pela Equação 1:

$$
\begin{aligned}
& \mathrm{Y}=\mathrm{b}_{\mathrm{o}}+\mathrm{b}_{1} \mathrm{~A}+\mathrm{b}_{2} \mathrm{~B}+\mathrm{b}_{3} \mathrm{C}+\mathrm{b}_{12} \mathrm{AB}+\mathrm{b}_{13} \mathrm{AC}+ \\
& \mathrm{b}_{23} \mathrm{BC}+\mathrm{b}_{123} \mathrm{ABC}
\end{aligned}
$$

onde: $\mathrm{Y}$ é a resposta, $\mathrm{b}_{\mathrm{o}}$ é a média dos efeitos na resposta $\mathrm{Y}$, $b_{\mathrm{i}}$ são os efeitos principais, $b_{\mathrm{ijk}}$ são os efeitos da interação entre os fatores A, B e C. O fator A é a concentração de pó de pneu, $\mathrm{B}$ é a concentração de peróxido e $\mathrm{C}$ é a concentração de co-agente.

\section{Resultados e Discussão}

Com o uso do planejamento fatorial de experimentos pretendeu-se avaliar a influência das variáveis de entrada (pó de pneu, peróxido de dicumila e bismaleimida), sobre os valores das variáveis resposta (tração, alongamento e inchamento em óleo) nas misturas vulcanizadas dinamicamente com PP/pó de pneu/SBR. Buscou-se definir as faixas ótimas de resposta, ou seja, faixas que maximizem os resultados. No total foram realizados nove experimentos para os valores reais dos níveis superior (+), inferior (-) e para o ponto central (0), com três réplicas para cada experimento, Tabela 1. A fração elastomérica do TPV foi mantida em 70 partes, assim o ponto médio da composição continha 50 partes de pó de pneu e 20 partes de SBR.

A Figura 1 mostra a variação de torque durante o processamento da mistura PP/Pó de pneu/SBR em misturador interno a $185{ }^{\circ} \mathrm{C}$. Nota-se que as misturas no nível superior de peróxido $(1,0 \mathrm{phr})$ apresentaram os maiores valores no torque final da mistura, independentemente do teor de elastômero virgem - SBR. O maior valor de torque en-

Tabela 1. Fatores e níveis utilizados no planejamento fatorial.

\begin{tabular}{lccc}
\hline \multicolumn{1}{c}{ Variáveis } & Nível - 1 & Nível 0 & Nível + 1 \\
\hline Pó de pneu, phr & 30 & 50 & 70 \\
BMI, phr & 0,5 & 1,75 & 3,0 \\
Peróxido, phr & 0,5 & 0,75 & 1,0 \\
\hline
\end{tabular}

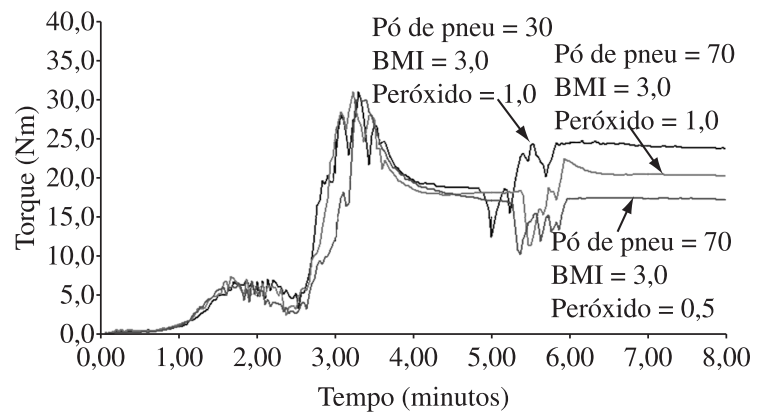

Figura 1. Processamento de TPV com pó de pneu em diferentes proporções. 
contrado foi para a composição na razão elastomérica de 40/30 (SBR/Pó de Pneu). Este comportamento sugere aumento na densidade de ligações cruzadas da mistura PP/Pó de Pneu/SBR, ou seja, há formação de novas reticulações na fase elastomérica, provavelmente da fase SBR. Ismail ${ }^{[8]}$ utilizou o Poli trans-octileno (TOR), um elastômero insaturado de baixo peso molecular, nas misturas de PP/pó de pneu. $\mathrm{O}$ autor atribuiu o aumento no torque de mistura a reticulação e ao efeito compatibilizante do TOR na mistura de PP/Pó de pneu. A adição de material virgem no composto produziu novas ligações que podem contribuir para o desempenho mecânico do TPV ${ }^{[6,8]}$. A composição nos níveis superiores de pó de pneu (70 phr) e de peróxido (1,0 phr), também apresentou aumento no torque final da mistura, este efeito pode ser atribuído ao aumento no nível de reticulação na fase pó de pneu, já curada. Entretanto, a mistura no nível superior de pó de pneu e no nível inferior de peróxido $(0,5 \mathrm{phr})$, não apresentou aumento no torque após a adição dos agentes de reticulação. Este comportamento pode ser arrogado a baixa quantidade de peróxido, insuficiente para modificar o torque de mistura.

A Tabela 2 apresenta a matriz planejamento com os valores médios dos diferentes experimentos realizados. Observa-se que os melhores resultados encontrados para a resistência à tração, alongmento e resistência ao inchamento em óleo, foram para as composições no nível superior de pó de pneu. Nota-se também aumento significativo da resistência à tração com o acréscimo do teor de peróxido nas composições. Atribui-se este resultado a compatibilização entre as fases, que ocorre durante o processamento dos materiais ${ }^{[9]}$. O sistema de reticulação a base de peróxido gera radicais livres tanto no polipropileno quanto no pó de pneu, esta reação reduz a massa molecular do PP e fragmenta o pó de pneu. O co-agente (BMI) é então atacado pelos macroradicais que formarão os copolímeros de PP-Pó de pneu. Esse copolímero é o responsável pelo aumento nas propriedades mecânicas do TPV a base de PP/Pó de pneu ${ }^{[9]}$. As misturas contendo material virgem ao contrário do esperado não apresentaram resultados satisfatórios para a resistência ao inchamento em óleo. Os valores obtidos foram próximos a 50\% de absorção de óleo no TPV. Nas misturas com SBR pode ocorrer a competição entre a formação do copolímero e a reticulação do SBR, provavelmente prevalece a formação de novas ligações na fase $\mathrm{SBR}^{[10]}$, que foi comprovado pelo aumento do torque de mistura, Figura 1. Esse comportamento pode ser provocado pela menor viscosidade do SBR em relação ao pó de pneu, que aumentaria a difusão do peróxido no elastômero não reticulado ${ }^{[8,10]}$. Porém, os melhores resultados de inchamento obtido foram para as misturas no nível superior de pó de pneu, ou seja, sem o SBR. Este comportamento pode ser atribuído a dois fatores: a formação do copolímero (PP-Pó de pneu) e/ou pelo aumento no percentual de carga neste TPV. As cargas reforçadoras como o negro de fumo, são utilizadas em formulações elastoméricas para aumentar as propriedades físico-mecânicas, além de reduzirem o inchamento dos compostos quando expostos a óleos e solventes ${ }^{[3]}$.

Os valores dos efeitos de cada parâmetro de resposta, tração, alongamento e inchamento em óleo dos TPVs foram calculados pelo programa estatístico MINITAB 13 . Os resultados obtidos do planejamento para a resposta de tração na ruptura considerando a ANOVA são apresentados na Tabela 3. Observa-se que os principais fatores (pó de pneu, BMI e peróxido) são estatisticamente significativos para a propriedade de tração na ruptura, pois os valores calculados de $\mathrm{F}$ são maiores que os valores críticos no limite de confiança de $95 \%$. Uma grande diferença entre $\mathrm{F}$ e $\mathrm{P}$ mostra que o efeito analisado é estatisticamente significativo ${ }^{[11,12]}$. No entanto o programa apresenta uma estatística t reportada para cada efeito, em vez da estatística aleatória F. O quadrado de uma variável aleatória t, com d graus de liberdade, é uma variável aleatória F, com 1 grau de liberdade no numerador e d graus de liberdade no denominador. Dessa forma, o quadrado da estatística t, será igual à estatística $\mathrm{F}^{[12]}$.

Dois fatores principais foram considerados significativos estatisticamente (pó de pneu e peróxido) para a propriedade de tração. O modelo obtido para o intervalo estudado foi:

$$
\text { Tração }=13,0796+0,6129 \mathrm{~A}+0,1529 \mathrm{C}
$$

onde: $\mathrm{A}$ = Pó de pneu; $\mathrm{B}=\mathrm{BMI}$; e $\mathrm{C}=$ Peróxido.

Os fatores principais contribuem positivamente para o aumento na tração, observa-se também que o fator A (pó de pneu) é mais importante que os fatores B e C. As interações

Tabela 2. Matriz planejamento com os valores obtidos nos diferentes experimentos.

\begin{tabular}{ccccccc}
\hline Pó de pneu & BMI & Peróxido & Tração $(\mathbf{M P a})$ & Alongamento $(\%)$ & Módulo $(\mathbf{M P a})$ & Inchamento em óleo $^{\mathbf{a}}(\%)$ \\
\hline+ & + & - & 14,85 & 116,60 & 75,0 & 21,00 \\
+ & - & - & 13,80 & 103,90 & 72,2 & 18,00 \\
- & - & - & 9,80 & 188,70 & 40,9 & 46,80 \\
- & - & + & 12,90 & 225,00 & 62,6 & 27,50 \\
+ & + & + & 13,82 & 206,60 & 68,2 & 34,00 \\
+ & - & + & 14,30 & 174,60 & 82,8 & 15,00 \\
- & + & - & 12,59 & 80,60 & 71,1 & 35,54 \\
0 & 0 & 0 & 14,20 & 180,10 & 73,4 & 20,15 \\
+ & + & + & 11,82 & 269,10 & 52,2 & 32,00 \\
\hline
\end{tabular}

anchamento em óleo de motor a $80^{\circ} \mathrm{C} / 72$ horas. 
Tabela 3. Análise do Minitab para a resposta de tração.

\begin{tabular}{|c|c|c|c|c|c|c|}
\hline \multicolumn{7}{|c|}{ Efeitos Estimados e coeficientes para tração } \\
\hline Termo & Efeito & Coeficiente & $\begin{array}{l}\text { Desvio padrão } \\
\text { do coeficiente }\end{array}$ & $\mathbf{T}$ & $\mathbf{P}$ & \\
\hline Constante & - & 13,0796 & 0,1996 & 65,54 & $<0,001$ & \\
\hline Pó de Pneu & 1,2258 & 0,6129 & 0,1996 & 6,74 & 0,007 & \\
\hline BMI & 0,7142 & 0,3571 & 0,1996 & 0,79 & 0,090 & \\
\hline Peróxido & 0,3058 & 0,1529 & 0,1996 & 2,95 & 0,006 & \\
\hline Pó de pneu*BMI & $-1,4292$ & $-0,7146$ & 0,1996 & $-3,58$ & 0,002 & \\
\hline Pó de pneu*peróxido & $-1,5762$ & $-0,7854$ & 0,1996 & $-6,67$ & $<0,001$ & \\
\hline BMI*peróxido & $-1,5392$ & $-0,7696$ & 0,1996 & $-3,86$ & 0,001 & \\
\hline Pódepneu*BMI*peróxido & $-0,2258$ & $-0,1129$ & 0,1996 & $-0,57$ & 0,579 & \\
\hline \multicolumn{7}{|c|}{ Análise de variância para a tração } \\
\hline Fonte & Graus de liberdade & SQ Seqüencial & SQ Ajustada & MQ Ajustada & $\mathbf{F}$ & $\mathbf{P}$ \\
\hline Efeitos principais & 3 & 12,6374 & 12,6374 & 4,2125 & 4,41 & 0,017 \\
\hline Interações de segunda ordem & 3 & 41,2774 & 41,2744 & 13,7581 & 14,39 & $<0,000$ \\
\hline Interações de terceira ordem & 1 & 0,306 & 0,3060 & 0,3060 & 0,32 & 0,579 \\
\hline Curvatura & 1 & 3,4479 & 3,4479 & 3,4479 & 3,61 & 0,074 \\
\hline Erro residual & 18 & 17,2069 & 17,2069 & 0,9559 & - & - \\
\hline Erro puro & 18 & 17,2069 & 17,2069 & 0,9559 & - & - \\
\hline Total & 26 & 74,8727 & - & - & - & - \\
\hline
\end{tabular}

de segunda ordem relatadas na Tabela 3, referem-se às interações entre os efeitos principais: $\mathrm{AB}, \mathrm{AC}, \mathrm{BC}$; e a interação de terceira ordem refere-se ao efeito ABC. Nota-se que só os efeitos principais têm significância estatística, altos valores de t.

Outra ferramenta que pode ser utilizada no estudo dos efeitos de resposta é a Metodologia de Superfície de Respostas (MSR) ${ }^{[12]}$. Na Figura 2 é ilustrado a superfície de resposta obtida para a tração em função dos fatores A e C. A curvatura da superfície mostra que o aumento do teor de pó de pneu e de peróxido contribui para o aumento da tração. Para maximizar a resposta da tração na região de 30 phr de pó de pneu é necessário o aumento na quantidade peróxido, entretanto essa alternativa eleva o custo do TPV. Que foge a proposta deste trabalho de obter um novo TPV com aplicação tecnológica, de baixo custo e com material reciclado.

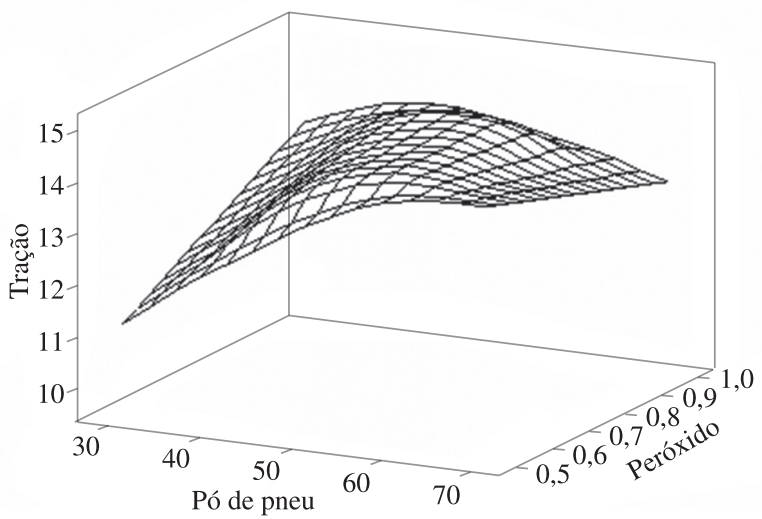

Figura 2. Superfície de resposta obtida para a tração em função dos fatores A e C.
Analisando os dados da Tabela 4, referentes à ANOVA para a resposta de alongamento na ruptura, observa-se que somente o fator $\mathrm{C}$ e a interação $\mathrm{AB}$, foram consideradas significativas. As interações de primeira ordem são mais relevantes que as demais, conforme os valores de $\mathrm{F}$ e $\mathrm{P}$. Ou seja, o fator preponderante do alongamento é a quantidade de peróxido adicionado ao material. O modelo obtido para o alongamento foi:

$$
\begin{aligned}
& \text { Alongamento na ruptura }=167,76+ \\
& 45,149 * \mathrm{C}+26,32 * \mathrm{AB}
\end{aligned}
$$

A Figura 3 apresenta o gráfico da resposta de superfície para o alongamento na ruptura em função do teor de pó de pneu e peróxido. Nota-se que o aumento na quantidade de peróxido duplica o valor do alongamento na ruptura. A técnica da vulcanização dinâmica aumenta o alongamento na

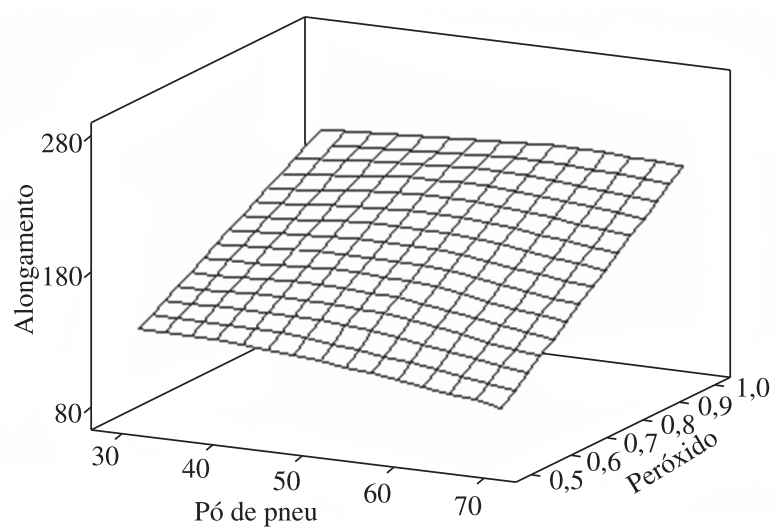

Figura 3. Superfície de reposta obtida para o alongamento na ruptura em função dos fatores $\mathrm{A}$ e $\mathrm{C}$. 
Tabela 4. Análise do Minitab para a resposta no alongamento.

\begin{tabular}{|c|c|c|c|c|c|c|}
\hline \multicolumn{7}{|c|}{ Efeitos estimados e coeficientes para alongamento } \\
\hline Termo & Efeito & Coeficiente. & $\begin{array}{l}\text { Desvio padrão } \\
\text { do coeficiente }\end{array}$ & $\mathbf{T}$ & $\mathbf{P}$ & \\
\hline Constante & - & 167,760 & 2,482 & 67,58 & $<0,000$ & \\
\hline Pó de Pneu & $-15,253$ & $-7,626$ & 2,482 & $-3,07$ & 0,007 & \\
\hline BMI & $-10,881$ & $-5,440$ & 2,482 & $-2,19$ & 0,042 & \\
\hline Peróxido & 90,298 & 45,149 & 2,482 & 18,19 & $<0,000$ & \\
\hline Pó de pneu*BMI & 52,648 & 26,324 & 2,482 & 10,60 & $<0,000$ & \\
\hline Pó de pneu*peróxido & 9,469 & 4,735 & 2,482 & 1,91 & 0,073 & \\
\hline BMI*peróxido & 37,098 & 4,735 & 2,482 & 1,91 & 0,073 & \\
\hline Pó de pneu*BMI*peróxido & $-8,031$ & $-4,015$ & 2,482 & $-1,62$ & 0,123 & \\
\hline \multicolumn{7}{|c|}{ Análise de variância para a tração } \\
\hline Fonte & Graus de liberdade & SQ Seqüencial & SQ Ajustada & MQ Ajustada & $\mathbf{F}$ & $\mathbf{P}$ \\
\hline Efeitos principais & 3 & 51028,0 & 51028,0 & 17009,3 & 115,01 & $<0,000$ \\
\hline Interações de segunda ordem & 3 & 25425,9 & 25452,9 & 8475,3 & 57,31 & $<0,000$ \\
\hline Interações de terceira ordem & 1 & 387,0 & 387,0 & 387,0 & 2,62 & 0,123 \\
\hline Curvatura & 1 & 406,1 & 406,1 & 406,1 & 2,75 & 0,115 \\
\hline Erro residual & 18 & 2662,1 & 2662,1 & 147,9 & - & - \\
\hline Erro puro & 18 & 2662,1 & 2662,1 & 147,9 & - & - \\
\hline Total & 26 & 79909,1 & - & - & - & - \\
\hline
\end{tabular}

Tabela 5. Análise do Minitab para a resposta no inchamento.

\begin{tabular}{|c|c|c|c|c|c|c|}
\hline \multicolumn{7}{|c|}{ Efeitos estimados e coeficientes para inchamento } \\
\hline Termo & Efeito & Coeficiente & $\begin{array}{c}\text { Desvio padrão } \\
\text { do coeficiente }\end{array}$ & $\mathbf{T}$ & $\mathbf{P}$ & \\
\hline Constante & - & 27,348 & 0,3473 & 78,74 & $<0,000$ & \\
\hline Pó de Pneu & $-11,858$ & $-5,929$ & 0,3473 & 17,07 & $<0,000$ & \\
\hline BMI & 1,412 & 0,706 & 0,3473 & 2,03 & 0,057 & \\
\hline Peróxido & $-0,442$ & $-0,221$ & 0,3473 & $-0,64$ & 0,533 & \\
\hline Pó de pneu*BMI & 8,425 & 4,212 & 0,3473 & 12,13 & $<0,000$ & \\
\hline Pó de pneu*peróxido & 4,612 & 2,306 & 0,3473 & 6,64 & $<0,000$ & \\
\hline BMI*peróxido & 10,342 & 5,171 & 0,3473 & 14,89 & $<0,000$ & \\
\hline Pó de pneu*BMI*peróxido & $-3,172$ & $-1,586$ & 0,3473 & $-4,57$ & $<0,000$ & \\
\hline \multicolumn{7}{|c|}{ Análise de variância para a tração } \\
\hline Fonte & Graus de liberdade & SQ Seqüencial & SQ Ajustada & MQ Ajustada & $\mathbf{F}$ & $\mathbf{P}$ \\
\hline Efeitos principais & 3 & 856,85 & 856,85 & 285,616 & 98,67 & $<0,000$ \\
\hline Interações de segunda ordem & 3 & 1195,19 & 1195,19 & 398,396 & 137,63 & $<0,000$ \\
\hline Interações de terceira ordem & 1 & 60,36 & 60,36 & 60,357 & 20,85 & $<0,000$ \\
\hline Curvatura & 1 & 131,82 & 131,82 & 131,820 & 45,54 & $<0,000$ \\
\hline Erro residual & 18 & 52,11 & 52,11 & 2,895 & - & - \\
\hline Erro puro & 18 & 52,11 & 52,11 & 2,895 & - & - \\
\hline Total & 26 & 2296,32 & - & - & - & - \\
\hline
\end{tabular}

ruptura dos TPV's, devido a distribuição da fase elastomérica reticulada na matriz termoplástica ${ }^{[13]}$.

Por meio da análise dos dados da Tabela 5, observa-se que as interações de segunda ordem são mais significativas que as de primeira ordem. Assim os fatores isolados não contribuem para melhorar a resistência ao inchamento. O pó de pneu contribui para reduzir o grau de inchamento do TPV, pois o valor do coeficiente calculado é negativo. O modelo obtido para o inchamento considerando as interações de segunda ordem, foi:

Inchamento $=27,348+5,171 \mathrm{BC}+$

$4,212 \mathrm{AB}+2,306 \mathrm{AC}$

A superfície de resposta para o inchamento é ilustrada na Figura 4. Nota-se que o aumento no teor de pó de pneu e peróxido reduz o inchamento do TPV. O gráfico da superfície de resposta apresenta curvatura característica de fatores com interações significativas. 


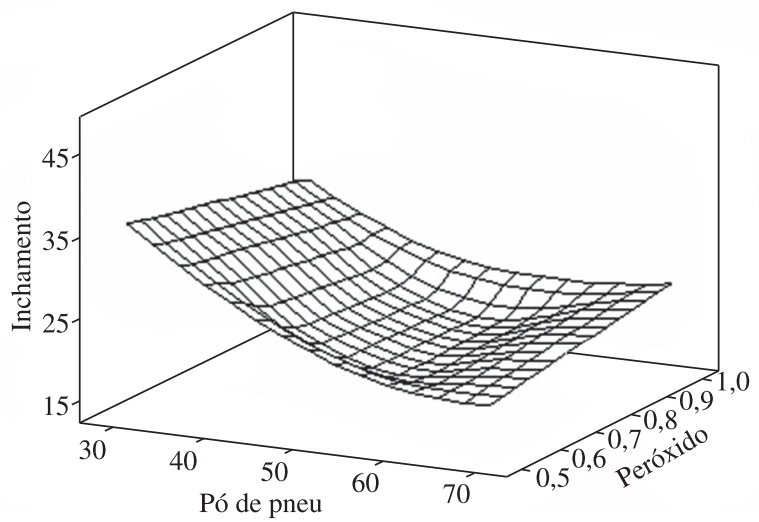

Figura 4. Superfície de reposta obtida para o inchamento em função dos fatores A e C.

\section{Conclusões}

- Os resultados evidenciam uma das principais vantagens da utilização do planejamento fatorial: a possibilidade de avaliar o sistema de maneira multivariada e co-relacionar todas as variáveis que compõem o sistema experimental.

- Os estudos conduzidos por planejamento fatorial de experimentos mostram que os efeitos do pó de pneu e do peróxido influenciam significativamente as propriedades avaliadas.

- Pode-se maximizar as propriedades do TPV com o uso de material reciclado como fase elastomérica.

- A resistência ao inchamento em óleo é maior com o aumento na quantidade de pó de pneu.

\section{Referências Bibliográficas}

1. Jang, J. W.; Yoo, T. S.; Oh, J. H. \& Iwasaki, I. - Conserv. Recycling, 22, p.1 (1998).
2. Pramanik, P. K. \& Baker, W. E. - Plast. Rubber Comp. Process Appl., 24, p.229 (1995).

3. Adhikari, B.; De, D. \& Maiti, S. - Prog. Polym. Sci., 25, p.909 (2000).

4. Kim, J. K. \& Burford R. P. - Rubber Chem. Technol., 71, p.1028 (1998).

5. Kumar, C. R., Fuhrmann, J. \& Kocsis, J. K. - Polym. Degrad. Stabil., 76, p.137 (2002).

6. Nakason, C., Wannavilai, P. \& Kaesaman A. - Polym. Test., 25, p.34 (2006).

7. Ismail, H. \& Suryadiansyah, A. - Polym. Plastics Technol. Eng., 45, p.463 (2006).

8. Awang, M., Ismail, H. \& Hazizan, M. A. - Polym. Test., 26, p.779 (2007).

9. Awang, M. \& Ismail, H. - Polym. Test., 27, p.321 (2008).

10. Dluzneski, P. R. - Rubber. Chem. Technol., 74, p.451 (2001).

11. Burns, R. E., Neto, B. B. \& Scarmínio I. S. - "Como fazer experimentos". Editora da Unicamp, Campinas (2001).

12. Montgomery, D. C. - "Estatística Aplicada e Probabilidade para Engenheiros", LTC, Rio de Janeiro, (2003).

13. Santos, D. M.; Sirqueira, A. S. \& Soares, B. G. - e-Polymer, p. 02 (2008).

Enviado: 05/02/09

Reenviado: 30/03/09

Aceito: $24 / 04 / 09$ 